geological literature of the world. We cannot find here lists of the publications of geological surveys; these are indexed under their authors and the districts with which the memoirs deal. Maps separately issued are not regarded as "literature"; but, under the heading "Maps," there is a very useful list of those included, and often concealed, in printed papers, with indications of their scales.

In connexion with the mechanism whereby pollen is able to induce hay fever, a correspondent has suggested that possibly the pollen grains in the presence of moisture on the mucous membranes might protrude their pollen tubes; these might penetrate the mucous membrane. A view somewhat similar was developed by Blackley half a century ago in his famous "Experimental researches on the causes and nature of Catarrhus aestivus." $\mathrm{He}$ showed that neither the size nor the nature of the covering of the pollen can be the essential cause of hay fever, but from prolonged observations he believed that the moisture on the mucous membrane might cause the pollen to swell and to protrude its tube into a mucous gland. While this might explain some of the initial phenomena in an attack of hay fever, he was strongly of opinion that the obnoxious element of pollen was the granular matter in the centre, a view universally accepted to-day.

\title{
Our Astronomical Cólumn.
}

LARGE Meteor.--In strong twilight on the evening of July ir a fine meteor was seen at $9^{\mathrm{h}} 9^{\mathrm{m}}$ G.M.T., by Mr. E. W. Barlow of Wadhurst, Sussex, who considered the object as bright as Venus at its best. The nucleus was pear-shaped and bluish, and a red train followed it along an arc of about $10^{\circ}$. The duration of flight was 4 seconds and the path from 95 Herculis to $\eta$ Ophiuchi.

Mr. E. H. Smith of Hanwell, W., also saw the object and describes the path with reference to the stars Altair and Antares. The height of the meteor was about 66-54 miles over the English Channel; length of path 90 miles, and velocity about 22 miles per second.

The fireball was also observed by the Astronomer Royal and by Dr. Crommelin at the Royal Observatory, Greenwich, and they give the azimuth of the end point as $15^{\circ} \mathrm{W}$. of south.

The Spiral Nebule as Dust-Clouds.-Mr. J. H. Reynolds discusses in Mon. Not. R.A.S. for May the recent suggestion of Prof. Lindemann that the spirals are dust-clouds expelled from the Galactic system by radiation pressure, and shining by reflected starlight. $\mathrm{He}$ gives a diagram of the distribution of the spirals, and of their radial velocities, determined by Prof. Slipher. The data as regards size, inclination, and radial velocity appear to fit in fairly well with Lindemann's theory, if one adopts the eccentric position of the sun in the Galaxy, as given by Prof. Shapley's determination of the distances of globular clusters. The spirals nearest to the Galactic centre would have the highest velocities, but would appear small to us, owing to distance. Those nearest to us would appear large, but would have small radial velocities, their motion being nearly across the line of sight. There is one feature of the spirals, however, that Mr. Reynolds regards as negativing the theory of their shining by reflected starlight. This is the dark absorption stripe which is seen to cross the centre of many of the spirals that are seen nearly edgewise. On the reflection hypothesis, this should be bright and not dark; its presence seems to prove that the illumination of the spirals comes from within them. Some years ago Mr. Reynolds put forward the view that the spirals were shining by reflecting the light of some bright body in their centre, basing this on measures of the relative brightness of different regions. $\mathrm{He}$ now repeats this suggestion, and adds that it may be possible for the condensed matter in the middle of the spiral to give a spectrum of type $F$ or $G$, without being in a stellar state. He quotes in support some recent experiments on the spectra given by exploded wires.
Stars in the Milky Way and at the Galactic PoLE.-The Harvard College Circ. No. 242 contains a very interesting comparison of a Milky Way field with one at the South Galactic Pole made by Mr. Solon I. Bailey. The aim of such investigations is not only to give an estimate of the total number of stars that exist or rather can be photographed, but also to form an idea of their distribution in space. Star gauges were made by the Herschels a century ago, but since then studies of the distributions of stars have been completed by Argelander, Seeliger, Pickering, Celoria, Kapteyn, Chapman and Melotte, and others. It was generally deduced that there were only twice as many stars in the Milky Way as elsewhere up to about magnitude ten, although Herschel's counts gave a maximum of about 20 times as many stars in the Galaxy as at the Galactic poles. An increasing Galactic concentration with decreasing apparent brightness was shown by Kapteyn, and a similar but less rapid degree of concentration was deduced by Chapman and Melotte. Mr. Bailey discusses photographs taken with the 24-inch Bruce photographic telescope at Arequipa, Peru, the longest exposures showing stars fainter than the nineteenth magnitude. He gives a very instructive table indicating the relation of length of exposure to limiting magnitudes photographed, showing that as the stars become fainter, longer and longer exposures have to be made to gain every extra magnitude. Selecting a square degree area in Sagittarius in the Galaxy, one of the richest star fields of the Milky Way, and comparing this with the similar area at the South Galactic Pole, he obtains the following counts :

\begin{tabular}{|c|c|c|}
\hline \multicolumn{2}{|c|}{ Exposures. } & Limiting \\
\hline $\mathrm{m}$ & s. & Magnitudes. \\
\hline 0 & I & IO.I \\
\hline O & $3 \cdot 3$ & $\mathrm{II} \cdot 2$ \\
\hline o & IO & $12 \cdot 4$ \\
\hline o & 30 & I $3 \cdot 5$ \\
\hline I & 29 & $I_{4} \cdot 6$ \\
\hline 4 & 27 & I $5 \cdot 6$ \\
\hline I3 & 20 & 16.5 \\
\hline 40 & $\mathrm{O}$ & $17 \cdot 4$ \\
\hline 120 & $\mathrm{O}$ & $18 \cdot 3$ \\
\hline 360 & o & $19 \cdot 2$ \\
\hline
\end{tabular}

$\begin{array}{rrr}\text { Galaxy. } & \text { Pole. } & \text { Ratio. } \\ \text { I3 } & 5 & 2 \cdot 6 \\ 47 & \text { I } 3 & 3 \cdot 6 \\ \text { II } & 29 & 3 \cdot 8 \\ 349 & 62 & 5 \cdot 6 \\ \text { I,945 } & \text { IO4 } & \text { I } 8 \cdot 7 \\ 9, \text { I60 } & \text { I5I } & 60 \cdot 7 \\ 2 I, 895 & 225 & 97 \cdot 3 \\ 36,260 & 359 & \text { IOI.0 } \\ 57, \text { I } 30 & 494 & \text { II } 5 \cdot 6 \\ 6 \text { I, 595 } & 55 \text { I } & \text { II I } \cdot 8\end{array}$

It will be seen that, up to about magnitude ro, the number of stars in the Milky Way is about 2.5 times that at the pole. After magnitude I3, the ratio increases with great rapidity, until at about the $17^{\text {th }}$ magnitude the ratio is more than roo. If the stars were everywhere as dense as in this part of the Galaxy their number would be 2.5 billions, while if the density were that at the pole, their number would be 23 millions.

$$
\text { NO. } 2803 \text {, vOL. I I } 2 \text { ] }
$$

